\title{
Regularity and propagation of moments in some nonlinear Vlasov systems
}

\author{
Ingenuin Gasser \\ Institut für Angewandte Mathematik, Universität Hamburg, \\ Bundesstraße 55, 20146 Hamburg, Germany, \\ Pierre-Emmanuel Jabin and \\ Benoit Perthame \\ Département de Mathématiques et d'Informatique, Ecole Normale Supérieure \\ 45 rue d'Ulm, 75230 Paris Cedex 05, France
}

\begin{abstract}
We introduce a new approach to prove the regularity of solutions to transport equations of the Vlasov type. Our approach is mainly based on the proof of propagation of velocity moments, as in a previous paper by Lions and Perthame [16]. We combine it with Moment Lemmas which assert that, locally in space, velocity moments can be gained from the kinetic equation itself. We apply our theory to two cases. First, to the Vlasov-Poisson system, and we solve a long standing conjecture, namely the propagation of any moment larger than two. Next, to the Vlasov-Stokes system where we prove the same result for fairly singular kernels.
\end{abstract}

\section{Introduction}

We consider the regularity of solutions to Vlasov sytems. These are nonlinear transport equations arising as the mean field limits of many-particles systems and are classical models arising for instance in plasma physics, astrophysics, 
fluid dynamics etc... Due to the nonlinearity, which arises because the force field acting on the particles depends on the density repartition of the particles themselves, these models exhibit a rather complex behavior. A particular example of this complexity is the difficulty to prove the regularity of solutions with smooth initial data.

We will describe our method on two examples of such systems. The first example is the famous Vlasov-Poisson system (VP in short). It describes the evolution of a density $f(x, v, t)$ of particles which at time $t \geq 0$, position $x \in \mathbb{R}^{3}$, move with the velocity $v \in \mathbb{R}^{3}$ and interact through self consistent coulombic or newtonian forces. It reads

$$
\begin{aligned}
& \frac{\partial}{\partial t} f+v \cdot \nabla_{x} f+\operatorname{div}_{v}(F f)=0 \\
& f(x, v, 0)=f^{0}(x, v) \geq 0
\end{aligned}
$$

with the force field

$$
F(t, x)= \pm \frac{x}{|x|^{3}} \star \rho(t, x),
$$

and, as usual, from the microscopic density $f$, we compute the macrospopic density $\rho$ and the current $j$ with the formulas

$$
\rho(x, t)=\int_{\mathbb{R}^{3}} f(x, v, t) d v, \quad j(x, t)=\int_{\mathbb{R}^{3}} v f(x, v, t) d v .
$$

The second example is the Vlasov-Stokes system (VS in short) which describes the evolution of particles interacting through a fluid described by a Stokes flow (see K. Hamdache [10] for another Vlasov-Stokes system, P.E. Jabin and B. Perthame [14] for the derivation of the system below from an interacting system of particles)

$$
\begin{gathered}
\frac{\partial}{\partial t} f+v \cdot \nabla_{x} f+\operatorname{div}_{v}[(F-v) f]=0, \\
f(x, v, 0)=f^{0}(x, v) \geq 0 \\
F(x, t)=A(x) \star j(x, t) .
\end{gathered}
$$

Here, the matrix $A \in C^{\infty}\left(\mathbb{R}^{3} \backslash 0\right)$ is assumed to satisfy two properties. The first property gives a limitation on the possible singularity at the origin, the second expresses the dissipation of the kinetic energy of the system (a natural condition since it is realized for the particle system),

$$
|A(x)| \leq \frac{C}{|x|^{\beta}}, \quad 0<\beta<2,
$$




$$
\int_{\mathbb{R}^{3}} j(x) \cdot A(x) \star j(x) d x \leq 0, \quad \forall j \in\left(\mathcal{D}\left(\mathbb{R}^{3}\right)\right)^{3} .
$$

For these two models, we are interested in the propagation of $v$-moments,

$$
M_{k}(t)=\sup _{0 \leq s \leq t} \int_{\mathbb{R}^{6}}|v|^{k} f(x, v, s) d v d x .
$$

Classical energy bounds (see [8], [13]) show that the second moment is a priori bounded

$$
M_{2}(t) \leq C\left(\left\|f^{0}\right\|_{\infty}, M_{2}(0)\right),
$$

where we denote by $\|u(\cdot)\|_{p}$ the $L^{p}$ norm of the function $u$ in its arguments $x$ or $(x, v)$ depending on the context.

Here, we prove the propagation of $v$-moments for $k$ larger than 2. As it is wellknown, this is a definitive step towards regularity of solutions because of the classical interpolation inequalities

$$
\begin{aligned}
& \|\rho(\cdot, t)\|_{(k+3) / 3} \leq C\|f(\cdot, \cdot, t)\|_{\infty}^{k /(3+k)} M_{k}(t)^{3 /(3+k)}, \\
& \|j(\cdot, t)\|_{(k+3) / 4} \leq C\|f(\cdot, \cdot, t)\|_{\infty}^{(k-1) /(3+k)} M_{k}(t)^{4 /(3+k)} .
\end{aligned}
$$

These inequalities, combined with Young (or generalized Young) inequalities furnish regularity for the force fields $F$.

$$
\begin{aligned}
& \left\|\frac{x}{|x|^{3}} \star \rho\right\|_{r} \leq C\|f(t)\|_{\infty}^{k /(k+3)} M_{k}(t)^{3 /(3+k)}, \quad r=3 \frac{3+k}{6-k}, \\
& \|A \star j\|_{r} \leq C\|f(t)\|_{\infty}^{(k-1) /(k+3)} M_{k}(t)^{4 /(3+k)}, \quad \frac{1}{r}=\frac{\beta}{3}+\frac{1-k}{k+3} .
\end{aligned}
$$

For $k$ large enough ( $k>6$ for the VP case, $k>3 \frac{\beta+1}{3-\beta}$ for the VS case), a control of $M_{k}$ therefore yields an $L^{\infty}$ bound on $F$ and thus allows to prove the propagation of the $(x, v)$ support of $f$, or of its derivatives, and thus to deduce its regularity.

Let us recall that the issue of the regularity of large solutions to nonlinear transport equations is a classical question still unresolved for several 3 dimensional models (Vlasov-Maxwell, Boltzmann). Several theories have been proposed for understanding the mechanisms that provide regularity. For the VP system, a method based on proving directly regularity through characteristics has been proposed by K. Pfaffelmoser [20], J. Batt [1], [2] and [3], J. Batt and G. Rein [6], J. Schaeffer[21], and E. Horst [11] and [12]. 
The case of 2D (or 2D and a half) Vlasov-Maxwell system is treated in R. Glassey and J. Schaeffer [9]. For the BGK-model of the Boltzmann equation, existence of smooth solutions follows from the control of propagation of the $L^{\infty}\left(\mathbb{R}^{6}\right)$ norms of $|v| f(\cdot, \cdot, t)$ (see B. Perthame and M. Pulvirenti [19]). For the Vlasov-Poisson Fokker-Planck system, still another theory has been developed by F. Bouchut [4].

Here, we will rather follow an approach based on proving the propagation of the velocity moments $M_{k}$, as in a previous paper by P.L. Lions and B. Perthame [16]. We combine it with Moment Lemmas which assert that, locally in space, velocity moments can be gained from the kinetic equation itself (see B. Perthame [17], P.L. Lions and B. Perthame [15], I. Gasser, P.A. Markowich and B. Perthame [7] or 2.2 below). This induces a difficulty in getting back global regularity in space despite the local aspect of the moment lemmas. We solve it using indirectly the propagation of $x$-moments of $f$. This method allows to simplify the method of [16], and to improve the results in the sense that we can prove the propagation of lower moments as we show it on the Vlasov-Poisson system, and also to handle stronger singularities in the nonlinearity as we illustrate it on the Vlasov-Stokes system. Namely, for the Vlasov-Poisson system, we prove the following result.

Theorem 1.1 (Vlasov-Poisson system) We assume that $f^{0} \in L^{\infty}\left(\mathbb{R}^{6}\right)$ and that, for some $k_{0}>2$, we have

$$
\begin{gathered}
\int_{\mathbb{R}^{6}}\left(1+|v|^{k_{0}}+|x|^{\frac{1}{3}+0}\right) f^{0}(x, v) d v d x<+\infty, \\
\rho^{0}(x, t):=\int_{\mathbb{R}^{3}} f^{0}(x-v t, v) d v \in L_{l o c}^{1}\left(0,+\infty ; L^{3\left(k_{0}+3\right) /\left(k_{0}+6\right)}\left(\mathbb{R}^{3}\right)\right) .
\end{gathered}
$$

Then, there exists a weak solution to (1.1), which satisfies for all $t, T>0$

$$
\begin{gathered}
f(x, v, t) \geq 0, \quad\|f(\cdot, \cdot, t)\|_{\infty} \leq\left\|f^{0}(\cdot, \cdot)\right\|_{\infty} \\
\int_{\mathbb{R}^{6}}\left(1+|v|^{k_{0}}+|x|^{\frac{1}{3}+0}\right) f(x, v, t) d v d x \in L^{\infty}(0, T), \\
F \in L^{1}\left(0, T ; L^{k_{0}+4-0}\left(\mathbb{R}^{3}\right)\right) \\
\rho \in L^{\infty}\left(0, T ; L^{\left(k_{0}+3\right) / 3}\left(\mathbb{R}^{3}\right)\right) .
\end{gathered}
$$




\section{Remarks}

1. Throughout this paper, when we use notations like $u \in L^{p+0}$, we mean that there exists an $\varepsilon>0$ such that $u \in L^{p+\varepsilon}$.

2. Let us notice that in the above theorem, we solve a question asked in [16]. Namely, to prove the propagation of a $v$-moment of order larger than 2 , while in [16] it is fundamental to control initially moments larger than 3.

3. Also, the regularity of the force field can be completed as follows

$$
\begin{gathered}
F \in L^{\infty}\left(0, T ; L^{r}\left(\mathbb{R}^{3}\right)\right), \\
r=3 \frac{k+3}{6-k} \text { for } 2<k<6, r=\infty \text { for } k>6 .
\end{gathered}
$$

4. An improvement is still possible. A careful application of the same proof shows that the assumption $f^{0} \in L^{\infty}$ can be relaxed to some $L^{p}$.

Turning now to the Vlasov-Stokes system, we prove the following result. It is the first regularity result for this system. The difficulty here comes from the lower $L^{p}$ regularity available on $j$ compared to $\rho$, and thus on the corresponding force $F$.

Theorem 1.2 (Vlasov-Stokes system) We assume that $0<\beta<8 / 5, f^{0} \in$ $L^{\infty}\left(\mathbb{R}^{6}\right)$ and that, for some $k_{0}>2$, we have

$$
\begin{gathered}
\int_{\mathbb{R}^{6}}\left(1+|v|^{k_{0}}+|x|^{2}\right) f^{0}(x, v) d v d x<+\infty, \\
J^{0}(x, t):=\int_{\mathbb{R}^{3}}|v| f^{0}(x-v t, v) d v \in L_{l o c}^{1}\left(0,+\infty ; L^{p}\left(\mathbb{R}^{3}\right)\right), \quad \frac{1}{p} \leq \frac{k_{0}+5}{k_{0}+4}-\frac{\beta}{3} .
\end{gathered}
$$

Then, there exists a weak solution to (1.4), which satisfies for all $t, T>0$

$$
\begin{gathered}
f(x, v, t) \geq 0, \quad\|f(\cdot, \cdot, t)\|_{\infty} \leq e^{3 t}\left\|f^{0}(\cdot, \cdot)\right\|_{\infty} \\
\int_{\mathbb{R}^{6}}\left(1+|v|^{k_{0}}+|x|^{2}\right) f(x, v, t) d v d x \in L^{\infty}(0, T), \\
F \in L^{1}\left(0, T ; L^{k_{0}+4-0}\left(\mathbb{R}^{3}\right)\right) \\
j \in L^{\infty}\left(0, T ; L^{\left(k_{0}+3\right) / 4}\left(\mathbb{R}^{3}\right)\right) .
\end{gathered}
$$




\section{Remarks}

1. Improving the possible singularity of the matrix $A$ i.e. the upper value of $\beta$ is an open question.

2. Again, the regularity of the force field can be completed as follows

$$
\begin{aligned}
F \in L^{\infty}\left(0, T ; L^{r}\left(\mathbb{R}^{3}\right)\right), & \frac{1}{r}=\frac{\beta}{3}+\frac{1-k}{k+3}, \text { for } 2<k<3 \frac{\beta+1}{3-\beta}, \\
r & =\infty, \text { for } k>3 \frac{\beta+1}{3-\beta} .
\end{aligned}
$$

The end of this paper explains the proof of these results. In a second section, we give the main lemmas and show the strategy of proof. The most fundamental estimate is specific to each case and its proof is detailed in separate sections.

\section{Proofs of the main Theorems}

In this section we are concerned with the proofs of the main Theorems (1.1) and (1.2). Before going to the new ingredients, we recall the general method and some necessary preliminary lemmas valid for both Vlasov-Poisson and Vlasov-Stokes system.

First of all, as usual to prove these theorems, we consider a sequence of classical solutions to a regularized system (with regularized positive convolution operators which define the forces, this is possible in truncating for high frequencies), with regularized and compactly supported initial data. It is enough to prove the estimates of the theorems for these solutions and then, to pass to the limit on the regularization. Secondly, for such solutions, the positivity and $L^{\infty}$ bounds stated in the theorems are true thanks to the maximum principle, as well as the kinetic energy bounds (see [8] or [13]) which can be kept by appropriate regularizations of the force kernel. The only difficult point is then to prove the propagation of moments higher than 2 . This proof follows the same lines for the two systems. But the form of the Vlasov Stokes system makes it longer due to the friction term which however does not add any specific difficulty. Therefore, we restrict our proof to the simplified system where we neglect the friction term i.e. we only consider the equation (1.1) with the two cases of forces $F$.

In the following, we set

$$
K_{\infty}=\left\|f^{0}\right\|_{\infty}
$$


and we recall some technical lemmas.

\subsection{Preliminary lemmas}

The first Lemma concerns the propagation of moments for solutions to the equation (1.1).

Lemma 2.1 Let $k>0$. Then, for $0 \leq t \leq T$, the moments $M_{k}(t)$, defined in (1.8), satisfy

$$
M_{k}(t) \leq C\left(T, K_{\infty}\right)\left(M_{k}(0)+\left(\int_{0}^{t}\|F(s)\|_{k+3} d s\right)^{k+3}\right) .
$$

This lemma is well known and can be proven easily using explicitely the Vlasov equation and the inequality

$$
\int_{\mathbb{R}^{3}}|v|^{k-1} f(x, v, t) d v \leq C\left(K_{\infty}\right)\left(\int_{\mathbb{R}^{3}}|v|^{k} f(x, v, t) d v\right)^{(k+2) /(k+3)},
$$

which generalizes (1.10).

The second result is a so called Moment Lemma about the gain of velocity moments by integration in time. It was first used in [17] to solve the BGK model. A more direct and systematic approach was deviced in [15]. The possibility to use it in order to control macroscopic quantitites was proved in [7].

Lemma 2.2 Let $\alpha>0, k \geq 1$ and $t>0$ and let $f$ be a smooth solution to the Vlasov equations (1.1), $\|F(.)\|_{k+3} \in L^{1}(0, T)$ and $M_{k}(t)<\infty$. Then, the inequality

$$
\begin{aligned}
\int_{0}^{t} \int_{\mathbb{R}^{6}} \frac{|v|^{k+1}}{1+|x|^{1+\alpha}} f(x, v, s) d x d v d s \leq & C\left(K_{\infty}\right)\left[M_{k}(t)\right. \\
& \left.+\int_{0}^{t}\|F(s)\|_{k+3} d s M_{k}(t)^{(k+2) /(k+3)}\right]
\end{aligned}
$$

holds for some constant which also depends upon $t, k, \alpha$. 
Proof. We multiply the Vlasov equation by

$$
|v|^{k-1} \frac{x \cdot v}{\left(1+|x|^{\alpha}\right)^{1 / \alpha}}, \quad \alpha>0, \quad k \geq 1,
$$

and integrate over $\mathbb{R}_{x}^{3} \times \mathbb{R}_{v}^{3} \times(0, t)$. After integration by parts and using (2.3), this yields

$$
\begin{aligned}
& \int_{0}^{t} \int_{\mathbb{R}^{6}} \frac{|v|^{k+1}}{\left(1+|x|^{\alpha}\right)^{1 / \alpha}}\left(1-\frac{|x|^{\alpha-2}(x \cdot v)^{2}}{\left(1+|x|^{\alpha}\right)|v|^{2}}\right) f(x, v, s) d x d v d s \\
\leq & \int_{\mathbb{R}^{6}}|v|^{k}(f(x, v, t)+f(x, v, 0)) d v d x \\
& +k \int_{0}^{t} \int_{\mathbb{R}_{x}^{3}}|F(x, s)|\left(\int_{\mathbb{R}_{v}^{3}}|v|^{k-1} f(x, v, s) d v\right) d x d s \\
\leq & 2\left(M_{k}(t)+C\left(K_{\infty}, k\right) \int_{0}^{t}\|F(s)\|_{k+3} d s M_{k}(t)^{(k+2) /(k+3)}\right) .
\end{aligned}
$$

Thanks to (2.3). Finally, we remark that

$$
\begin{aligned}
& \int_{0}^{t} \int_{\mathbb{R}^{6}} \frac{|v|^{k+1}}{1+|x|^{1+\alpha}} f(x, v, s) d x d v d s \\
\leq & C(\alpha) \int_{0}^{t} \int_{\mathbb{R}^{6}} \frac{|v|^{k+1}}{\left(1+|x|^{\alpha}\right)^{1 / \alpha}}\left(1-\frac{|x|^{\alpha-2}(x \cdot v)^{2}}{\left(1+|x|^{\alpha}\right)|v|^{2}}\right) f(x, v, s) d x d v d s,
\end{aligned}
$$

which concludes the result.

\subsection{Another formula for the force fields}

The following result is the main new ingredient in the proofs of the Theorems. It improves the method introduced in the analysis of the Vlasov-Poisson system by [16] in order to use the moment Lemma 2.2. Since the exponents coming in for the two systems are quite different, we state the result separately.

Lemma 2.3 (VP system) Smooth solutions to the regularized Vlasov Poisson system (1.1) (see above) satisfy

$$
\begin{aligned}
\int_{0}^{T}\|F(t)\|_{r} d t \leq & C(T) \int_{0}^{T}\left\|\rho^{0}(t)\right\|_{p} d t \\
& +C_{1}\left(\int_{0}^{T} \int_{\mathbb{R}^{6}} \frac{|v|^{k+1}}{1+|x|^{1+0}} f(x, v, t) d x d v d t\right)^{1 / r},
\end{aligned}
$$


for all $3<r<k+4$ and with $\frac{1}{r}=\frac{1}{p}-\frac{1}{3}$ and where $C_{1}$ also depends on the parameters $k, r$ and $K_{\infty}$, the initial energy $M_{2}(0)$ and $\int_{\mathbb{R}^{6}}|x|^{\frac{1}{3}+0} f^{0}(x, v) d x d v$.

Lemma 2.4 (VS system) Smooth solutions to the regularized Vlasov Stokes system (1.4) (see above) satisfy

$$
\begin{aligned}
\int_{0}^{T}\|F(t)\|_{r} d t \leq & C(T) \int_{0}^{T}\left\|J^{0}(t)\right\|_{p} d t \\
& +C_{2} \int_{0}^{T} \int_{\mathbb{R}^{6}}\left(\frac{|v|^{k+1}}{1+|x|^{1+0}} f(x, v, t) d x d v d t\right)^{1 / r}
\end{aligned}
$$

for all $r$ such that $\frac{1}{k+1}+\frac{\beta-2}{3}<\frac{1}{r}<\frac{\beta-1}{3}$ and with $\frac{1}{r}>\frac{1}{p}+\frac{\beta-3}{3}$. Here $C_{2}$ also depends on the parameters $\beta, p, k, r$ and $K_{\infty}$, the initial kinetic energy $M_{2}(0)$ and $\int_{\mathbb{R}^{6}}|x|^{2} f^{0}(x, v) d x d v$.

The proofs of these lemmas are given in the next section. With these three types of lemmas, we are now able to prove our main theorem.

\subsection{Concluding the proofs of the main theorems}

\subsubsection{Vlasov-Poisson case}

We combine the lemmas 2.3 and 2.2 so as to get

$$
\begin{aligned}
\int_{0}^{T}\|F(t)\|_{r} d t \leq & C \int_{0}^{T}\left\|\rho^{0}(t)\right\|_{p} d t \\
& +C\left(M_{k}(T)+M_{k}(t)^{(k+2) /(k+3)} \int_{0}^{T}\|F(t)\|_{k+3} d t\right)^{1 / r},
\end{aligned}
$$

with $3<r<k+4$, and $\frac{1}{r}=\frac{1}{p}-\frac{1}{3}$.

The assumption on $\rho^{0}$ in the theorem 1.1 allows us to control the integral of $\left\|\rho^{0}\right\|_{p}$ for all corresponding $\mathrm{r}$ between 3 and $k_{0}+3$ included. We already know that the kinetic energy $M_{2}$ is bounded in time and thus we can apply the result (2.9) for $k=2$ (recall we already control $F$ in $L_{t}^{1} L_{x}^{p}$ for all $p<5$ thanks to (2.7)), thus obtaining that the integral in time of the $L^{r}$ norm of $F$ is bounded for any $3<r<6$.

As a consequence, using lemma 2.1 , we immediately propagate every moment $2<k<3$, thus concluding the theorem for $k_{0}<3$. Then, for $k_{0} \geq 3$, we repeat the above argument using any $k<3$, which allows, with (2.9), to 
reach $r<7$ and therefore, using lemma 2.1 again, to propagate any moments up to $k<4$, thus concluding the theorem for $k_{0}<4$. One can easily see that each repetition of these two steps allows to gain one order on $k$ for the propagation of moments, and we are thus able to reach any value $k_{0}$.

\subsubsection{The Vlasov-Stokes case}

The proof for Vlasov-Stokes system follows the same lines. We use lemmas 2.4 and 2.2 to obtain

$$
\begin{aligned}
\int_{0}^{T}\|F(t)\|_{r} d t \leq & C \int_{0}^{T}\left\|J^{0}\right\|_{p} d t \\
& +C\left(M_{k}(T)+M_{k}(t)^{(k+2) /(k+3)} \int_{0}^{T}\|F(t)\|_{k+3} d t\right)^{1 / r},
\end{aligned}
$$

with $\frac{3}{\beta-1}<r<\left(\frac{1}{k+1}-\frac{2-\beta}{3}\right)^{-1}$ and $\frac{1}{p}<\frac{1}{r}+\frac{3-\beta}{3}$.

Like for the VP case, the assumption on $J^{0}$ in the theorem is enough to upper bound the $J^{0}$ term in the above inequality (2.10), and this for all for $\frac{3}{\beta-1}<r \leq k_{0}+3$. First, we use, from the energy, that $M_{2}$ is bounded, and from (2.8), we deduce that $F$ is bounded in $L_{t}^{1} L_{x}^{p}$ for all $p<5$. And thus, from the inequality (2.10), we get that $F$ belongs to $L_{t}^{1} L_{x}^{r}, r$ being given by the above formula, and, using lemma 2.1 , we propagate every moment of order $k^{\prime}$ less than $r-3$ i.e.,

$$
\frac{1}{k^{\prime}+3}>\frac{1}{k+1}-\frac{2-\beta}{3} .
$$

For $k=2$ this allows to control moments with $k^{\prime}>2$ (this explains the limitation $\beta<8 / 5)$. Moreover, iterating the argument, for $k$ larger or equal to $2, k^{\prime}$ can be chosen strictly larger (with a uniform gap) than $k$ because the $L_{x}^{k+3}$ bound on $F$ is then automatic. And we can repeat this procedure until we propagate all the desired moments thus concluding the proof.

\section{Estimates on the force fields}

In this section, we prove the fundamental lemmas 2.3 and 2.4 stated in the previous section and which allow to obtain a better $L^{r}$ estimate on the force field, working in $L^{1}$ in time rather than $L^{\infty}$, and using some kind of localisation in space. The exponents arising in the proof depend on the specific form 
of the force fields and therefore differ somewhat in the two cases of Poisson and Stokes flows. The proofs are thus presented in two subsections.

They both use the following common expression for the Vlasov equation (recall that we neglect the friction term in the Stokes case to simplify the proofs)

$$
\frac{\partial}{\partial t} f+v \cdot \nabla_{x} f+\operatorname{div}_{v}\left(F_{L} f\right)=\operatorname{div}_{v}\left(F_{S} f\right),
$$

where the long range part of the force is given by $F_{L}=F_{S}+F$, and the short range part of the force is defined for the VP system as $F_{S}:= \pm \rho \star\left(\chi_{R} \frac{x}{|x|^{3}}\right)$, and for the VS system as $F_{S}:=j \star\left(\chi_{R} A\right)$, where $\chi_{R}$ is a smooth cut-off function of the ball of radius $R$, which vanishes for $|x|>2 R$ and such that $\chi_{R}(x)=1$ for $|x| \leq R$. We also use a representation of the solution related to the well defined characteristics

$$
\begin{aligned}
& \frac{d}{d t} X(t)=V(t), \quad X(0)=x, \\
& \frac{d}{d t} V(t)=F_{L}(t, X(t)), \quad V(0)=v .
\end{aligned}
$$

We now choose the truncation parameter $R$ large enough compared to the final time $T$, so that these characteristics and their partial jacobians, behave like $X(t)=x-v t, V(t)=v$ which are obtained for $F_{L}=0$ i.e. the limit as $R \rightarrow \infty$. For the sake of simplicity, the proofs below are written for these limiting characteristics, but the arguments hold for those given by (3.2) as it was checked in [16], the numerous changes of variables only require to control jacobians of $\frac{\partial X}{\partial x}, \frac{\partial X}{\partial v} \ldots$

With this simplification, we have

$$
\begin{aligned}
f(x, v, t)= & f^{0}(x-v t, v)+\int_{0}^{t}\left(\operatorname{div}_{v} F_{S} f\right)(x-v s, v, t-s) d s \\
= & f^{0}(x-v t, v)+\int_{0}^{t} \operatorname{div}_{v}\left(F_{S}\right) f(x-v s, v, t-s) d s \\
& +\int_{0}^{t} \operatorname{div}_{x}\left(F_{S}\right) f(x-v s, v, t-s) s d s .
\end{aligned}
$$

\subsection{The Vlasov Poisson System}

Now, we restrict our attention to the Vlasov-Poisson case. From the above formula, we deduce,

$$
\rho(x, t)=\int_{\mathbb{R}^{3}} f^{0}(x-v t, v) d v+\int_{0}^{t} \int_{\mathbb{R}^{3}} \operatorname{div}_{x}\left(F_{S} f\right)(x-v s, v, t-s) s d s d v .
$$


Using that $F$ wins a full derivative compared to $\rho$ in $L^{p}$ norms, we deduce, from Calderon-Zygmung theory (see [22]), with $1 / r=1 / p-1 / 3$ and recalling the definition of $\rho^{0}$ in the Theorem 1.1,

$$
\|F(\cdot, t)\|_{r} \leq\left\|\rho^{0}(\cdot, t)\right\|_{p}+\int_{0}^{t}\left\|\int_{\mathbb{R}^{3}}\left(F_{S} f\right)(x-v s, v, t-s) d v\right\|_{r} s d s .
$$

We now treat the second $L^{r}\left(\mathbb{R}_{x}^{3}\right)$ norm term in a new way. We write, $x$ being fixed, using Holder inequality in $d v$,

$$
\begin{aligned}
\mid \int\left(F_{S} f\right)(x & -v s, v, t-s) d v \mid \\
& \leq\left\|F_{S}(x-v s, t-s) \mid\left(1+|x-v s|^{\frac{1}{3}+0}\right)\right\|_{\frac{3}{2}+0} \\
& \times\left\|f(x-v s, v, t-s)\left(1+|x-v s|^{\frac{1}{3}+0}\right)^{-1}\right\|_{3-0} \\
& \leq K_{\infty}^{\frac{2}{3}-0} \frac{1}{s^{2-0}}\left\|F_{S}(\cdot, t-s) \mid\left(1+|\cdot|^{\frac{1}{3}+0}\right)\right\|_{\frac{3}{2}+0} \\
& \times\left(\int_{\mathbb{R}^{3}} f(x-v s, v, t-s) \times\left(1+|x-v s|^{1+0}\right)^{-1} d v\right)^{\frac{1}{3}-0} \\
& \leq K_{\infty}^{\frac{2}{3}+\frac{k+1}{k+4}-0} \frac{1}{s^{2-0}}\left\|F_{S}(\cdot, t-s)\left(1+\left.|\cdot|\right|^{\frac{1}{3}+0}\right)\right\|_{\frac{3}{2}+0} \\
& \times\left(\int_{\mathbb{R}^{3}}|v|^{k+1} f(x-v s, v, t-s)\right. \\
& \left.\times\left(1+|x-v s|^{1+0}\right)^{-1} d v\right)^{\frac{1}{4+k}-0},
\end{aligned}
$$

here we have used a variant of the general interpolation inequality (2.3). We now conclude, using $r=4+k-0$ that

$$
\begin{aligned}
\|F(\cdot, t)\|_{r} & \leq\left\|\rho^{0}(\cdot, t)\right\|_{p}+C\left(K_{\infty}\right) \int_{0}^{t}\left\|F_{S}(\cdot, t-s)\left(1+|\cdot|^{\frac{1}{3}+0}\right)\right\|_{\frac{3}{2}+0} \\
& \times\left(\int_{\mathbb{R}^{6}}|v|^{k+1} f(x-v s, v, t-s)\left(1+|x-v s|^{1+0}\right)^{-1} d v d x\right)^{1 / r} \frac{d s}{s^{1-0}} .
\end{aligned}
$$

After integrating in time and changing the variable $d x=d(x-v s)$ we obtain the lemma 2.3. Indeed, the mass and energy propagations imply that

$$
\begin{gathered}
\left|F_{S}(x, \sigma)\right|\left(1+|x|^{\alpha}\right) \mid \leq C \int_{|x-y| \leq R} \frac{\rho(y, \sigma)}{|x-y|^{2}}\left(1+|x-y|^{\alpha}+|y|^{\alpha}\right) \\
\leq C \rho \star \frac{1}{|x|^{2}}+C \rho \star \frac{1}{|x|^{2-\alpha}}+C\left(|x|^{\alpha} \rho \star \frac{1}{|x|^{2}}\right) .
\end{gathered}
$$

For $0 \leq \alpha<2$, each of these terms is bounded in $L^{1} \cap L^{s}$ for some $s>3 / 2$ thanks to the a priori estimates $\rho \in L^{\infty}\left(0, \infty ; L^{1}\right)$ (mass conservation), $\rho \in$ $L^{\infty}\left(0, \infty ; L^{5 / 3}\right)$ (energy conservation and $\left.(1.10)\right)$, and $|x|^{\alpha} \rho \in L^{\infty}\left(0, \infty ; L^{1} \cap\right.$ $L^{s}$ ) (propagation of $x$-moments of order less than 2 and interpolation with $\left.\rho \in L^{\infty}\left(0, \infty ; L^{5 / 3}\right)\right)$. 


\subsection{The Vlasov Stokes system}

The proof of lemma 2.4 for the Vlasov-Stokes system follows the same ideas as for the Vlasov Poisson system. First of all, in place of (3.2) we use the characteristics defined by the regular force term which is the sum of the friction term and the long range part of the force $F(t, x)$ to find the estimate (see the beginning of section 3 for notations)

$$
\begin{aligned}
\int_{0}^{T}\|F(., t)\|_{r} d t \leq & \int_{0}^{T}\left\|J^{0}(t)\right\|_{p} d t \\
& +\int_{0}^{T}\left\|\int_{0}^{t} \int_{\mathbb{R}^{3}} F_{S} f(x-v s, v, t-s) d v d s\right\|_{a} d t \\
& +\int_{0}^{T}\left\|\int _ { 0 } ^ { t } s \int _ { \mathbb { R } ^ { 3 } } \left|v\left\|F_{S} \mid f(x-v s, v, t-s) d v d s\right\|_{b} d t,\right.\right.
\end{aligned}
$$

with the relations

$$
\frac{1}{r}=\frac{1}{a}+\frac{\beta}{3}-1=\frac{1}{b}+\frac{\beta-2}{3},
$$

We denote

$$
\begin{aligned}
& I=\int_{0}^{T}\left\|\int_{0}^{t} \int_{\mathbb{R}^{3}} F_{S} f(x-v s, v, t-s) d v d s\right\|_{a} d t \\
& I I=\int_{0}^{T}\left\|\int _ { 0 } ^ { t } s \int _ { \mathbb { R } ^ { 3 } } \left|v\left\|F_{S} \mid f(x-v s, v, t-s) d v d s\right\|_{b} d t .\right.\right.
\end{aligned}
$$

In the next two subsections, we explain how we can upperbound these two terms.

\subsubsection{Bound on the term $I$ in the inequality (3.6)}

We write

$$
I \leq \int_{0}^{T}\left\|\int_{0}^{t}\right\| F_{S}(. .)\left\|_{L_{v}^{c}} \times\right\| f(. .)\left\|_{L_{v}^{\star}} d s\right\|_{L_{x}^{a}} d t,
$$

where $c^{\star}$ is the conjugate exponent of $c$ and with

$$
\left\|F_{S}(x-v s, t-s)\right\|_{L_{v}^{c}}=s^{-3 / c}\left\|F_{S}(., t-s)\right\|_{L_{x}^{c}} \leq C s^{-3 / c} \text {, for } c>3 .
$$

Indeed the conservation of the kinetic energy implies that $j$ belongs to $L^{1} \cap L^{5 / 4}\left(\mathbb{R}^{3}\right)$ and hence we already know that $F_{S}$ belongs to $L^{3 / \beta} \cap L^{15 /(5 \beta-3)}$. For $1 \leq \beta<8 / 5$, we have $15 / 8<\frac{3}{\beta} \leq 3$ and $3<\frac{15}{5 \beta-3} \leq 15 / 2$. Therefore the force term $F_{S}$ always belongs to $L^{\infty}\left([0, T], L^{c}\left(\mathbb{R}^{3}\right)\right)$ for some $c>3$. 
Next, we recall that

$$
\int_{\mathbb{R}^{3}} f(x-v s, v, t-s) d v \leq C K_{\infty}\left(\int|v|^{\delta} f(x-v s, v, t-s) d v\right)^{3 /(3+\delta)} .
$$

Combining the last two inequalities, for any $\frac{3}{2}<a<\frac{5}{2}$,

$$
\begin{aligned}
I & \leq C \int_{0}^{T}\left\|\int_{0}^{t} s^{-3 / c}\right\| f(x-v s, v, t-s)\left\|_{L_{v}^{c^{\star}}} d s\right\|_{L_{x}^{a}} d t \\
& \leq C \int_{0}^{T} \int_{0}^{t} s^{-3 / c}\left\|\left(\int f(x-v s, v, t-s) d v\right)^{1 / c^{\star}}\right\|_{L_{x}^{a}} d s d t \\
& \leq C \int_{0}^{T} \int_{0}^{t} s^{-3 / c}\left(\int|v|^{\delta} f d v d x\right)^{1 / a} d s d t \\
& \leq C,
\end{aligned}
$$

since we have $\frac{3+\delta}{3}=\frac{a}{c}$, and so $\delta$ is less than 2 , for $a$ is less than $\frac{5}{2}$ and $c^{\star}$ is less than $\frac{3}{2}$ but as close to $\frac{3}{2}$ as we want.

Sinece the system conserves mass and kinetic energy, it also conserves all the moments between 0 and 2 in velocity. Eventually, we have proved that $\int_{0}^{T}\left\|\int_{0}^{t} \int_{\mathbb{R}^{3}}\left|F_{S}\right| f(x-v s, v, t-s) d v d s\right\|_{a} d t$ is bounded for all $a$ between $\frac{3}{2}$ and $\frac{5}{2}$ included. Using relation (3.7), this can be used for any $r$ between $3 /(\beta-1)$ and $+\infty$ since $\beta$ is less than $\frac{9}{5}$ (we work with $\beta$ less than $\frac{8}{5}$ ).

\subsubsection{Bound on the term $I I$ in the estimate (3.6)}

First, we perform the same manipulation as in the previous section

$$
\begin{aligned}
I I \leq & \int_{0}^{T}\left\|\int_{0}^{t}\right\|(1+|x-v s|) F_{S}(x-v s, t-s) \|_{L_{v}^{d}} \\
& \times\left\|\frac{v f(x-v s, v, t-s)}{1+|x-v s|}\right\|_{L_{v}^{d^{\star}}} d s \|_{L_{x}^{b}} d t .
\end{aligned}
$$

We choose for $d$ a number slightly greater than $\frac{3}{2}$ and we bound the term with $F_{S}$ by $s^{-3 / d}\left\|(1+|x|) F_{S}(x, t-s)\right\|_{d}$ and we decompose this last term as

$$
\begin{aligned}
\left|(1+|x|) F_{S}(x, t-s)\right| \leq & \int(1+|x|) \frac{\chi_{R}(x-y)}{|x-y|^{\beta}}|j(y)| d y \\
\leq & \int \frac{\chi_{R}(x-y)}{\left.|x-y|\right|^{\beta}} j(y) d y \\
& +C \int \frac{\chi(x-y)}{|x-y|^{\beta-1}} j(y) d y \\
& +C \int \frac{\chi_{R}(x-y)}{|x-y|^{\beta}}|y| j(y) d y .
\end{aligned}
$$

For $\beta$ less than 2 , the first two terms on the right handside are obviously in $L^{d}$ for some $d$ larger than $\frac{3}{2}$ but as close as we wish. To bound the last term in $L^{d}$, we need an $L^{1}$ estimate on $|y| j(y)$ which is given by the following lemma 
Lemma 3.1 If the kinetic energy is bounded, then the equation (1.4) conserves all the moments in $\int|x|^{\delta} \rho(x, t) d x$ for $\delta$ between 0 and 2.

Proof. This lemma is a straightforward consequence of the simple relation

$$
\frac{d}{d t} \int_{\mathbb{R}^{3}}(1+|x|)^{\delta} \rho(x, t) d x=\int_{\mathbb{R}^{6}} \delta(1+|x|)^{\delta-1} \frac{x}{|x|} \cdot v f(x, v, t) d x d v .
$$

Thanks to this lemma, we know that $\int|x|^{2} \rho(x, t) d x$ belongs to $L^{\infty}([0, T])$ and thus $\int|x| \cdot|j(x, t)| d x$ also because of the inequality

$$
\int_{\mathbb{R}^{3}}|x| \cdot|j(x, t)| d x \leq \frac{1}{2} \int_{\mathbb{R}^{6}}|v|^{2} f d x d v+\frac{1}{2} \int_{\mathbb{R}^{3}}|x|^{2} \rho(x, t) d x .
$$

As a consequence for any $d$ greater than $\frac{3}{2}$ but close enough, we have

$$
\left\|(1+|x-v s|) F_{S}(. .)\right\|_{L_{v}^{d}} \in L^{\infty}\left([0, T] \times \mathbb{R}^{3}\right) .
$$

We immediately deduce that

$$
\begin{aligned}
I I & \leq C \int_{0}^{T} \int_{0}^{t} s^{1-\frac{3}{d}}\left\|\frac{v f(x-v s, v, t-s)}{1+|x-v s|}\right\|_{L_{x}^{b}\left(L_{v}^{d^{\star}}\right)} d s d t \\
& \leq C \int_{0}^{T} \int_{0}^{t} s^{1-\frac{3}{d}}\left\|\left(\int \frac{\left.|v|\right|^{\star} f(x-v s, v, t-s)}{(1+|x-v s|)^{d^{\star}}} d v\right)^{1 / d^{\star}}\right\|_{b} d s d t,
\end{aligned}
$$

using the inequality

$$
\int_{\mathbb{R}^{3}} \frac{|v|^{\alpha} f(x-v s, v, t-s)}{(1+|x-v s|)^{\delta}} d v \leq \leq C\left(\int_{\mathbb{R}^{3}} \frac{|v|^{\gamma} f(. .)}{(1+|x-v s|)^{\delta}} d v\right)^{(3+\alpha) /(3+\gamma)}
$$

Then recalling that $d=\frac{3}{2}+0$, if $b$ is greater than $d^{\star}$ we find

$$
\begin{aligned}
I I & \leq C \int_{0}^{T} \int_{0}^{t} s^{1-\frac{3}{d}}\left(\int_{\mathbb{R}^{6}} \frac{|v|^{\delta} f(x-v s, v, t-s)}{(1+|x-v s|)^{d^{\star}}} d v d x\right)^{1 / b} d s d t \\
& \leq C \int_{0}^{T} \int_{0}^{t} s^{1-\frac{3}{d}}\left(\int_{\mathbb{R}^{6}} \frac{|v|^{\delta} f(x, v, t-s)}{(1+|x|)^{\star}} d v d x\right)^{1 / b} d s d t \\
& \leq C \int_{0}^{T} \int_{s}^{T} s^{1-\frac{3}{d}}\left(\int_{\mathbb{R}^{6}} \frac{|v|^{\delta} f(x, v, t-s)}{(1+|x|)^{d^{\star}}} d v d x\right)^{1 / b} d t d s \\
& \leq \tilde{C} \int_{0}^{T}\left(\int_{\mathbb{R}^{6}} \frac{|v|^{\delta} f(x, v, t)}{(1+|x|)^{d^{\star}}} d v d x\right)^{1 / b} d t \\
& \leq C^{\prime}\left(\int_{0}^{T} \int_{\mathbb{R}^{6}} \frac{\left.|v|\right|^{\delta} f(x, v, t)}{(1+|x|)^{d^{\star}}} d v d x d t\right)^{1 / b}
\end{aligned}
$$


with the relation $\frac{d^{\star}}{b}=\frac{3+d^{\star}}{3+\delta}$. Since $d^{\star}=3-0$, if we denote $k=\delta-1$ this implies

$$
k=b-1+0 .
$$

We can now conclude the proof. The result from section 3.2.1 is valid for $r$ between $3 /(\beta-1)$ and $+\infty$, and the result from section 3.2.2 for $b$ larger than $3-0$ which means $r$ larger than $3 /(\beta-1)$ thanks to relation (3.7). Hence for $r$ larger than $3 /(\beta-1)$, we can put together these two results and get

$$
\begin{aligned}
\int_{0}^{T}\|F(., t)\|_{r} d t \leq & \int_{0}^{T}\left\|J^{0}\right\|_{p} d t+C( \\
& \left.1+\left(\int_{0}^{T} \int_{\mathbb{R}^{6}} \frac{|v|^{k+1} f(x, v, t)}{(1+|x|)^{d^{\star}}} d v d x d t\right)^{1 / b}\right),
\end{aligned}
$$

with

$$
\frac{1}{b}=\frac{1}{r}+\frac{2-\beta}{3}, \quad k=b-1+0,
$$

which is exactly the lemma 2.4.

Acknowledgements. The first author acknowledges support from the european TMR network Asymptotic Methods in Kinetic Theory and from the PROCOPE project entitled Verallgemeinerte Halbleitermodelle funded by the german DAAD.

\section{References}

[1] J. Batt, Global symmetric solutions of the initial value problem of stellar dynamics, J. Diff. Eq., 25, pp 342-364, 1977.

[2] J. Batt, The non-linear Vlasov-Poisson system of partial differential equations in stellar dynamics, Publ. CNER Math. Pures Appl., 5, pp 1-30, 1983.

[3] J. Batt, The present state of the existence theory of the Vlasov-Poisson and Vlasov-Maxwell system of partial differential equations in plasma physics, in V. Boffi, H. Neunzert, Applications of mathematics in technology, Proceedings Rome 1984, pp 375-385, Stuttgart: Teubner 1984. 
[4] F. Bouchut, Smoothing effect for the non-linear Vlasov-Poisson-FokkerPlanck system, J. Diff. Eq., 122, pp 225-238, 1995.

[5] F. Bouchut, Existence and uniqueness of a global smooth solution for the Vlasov-Poisson-Fokker-Planck system in three dimensions, J. Funct. Anal., 111, pp 239-258, 1993.

[6] J. Batt, G. Rein, Global classical solutions of the periodic VlasovPoisson system in three dimensions, C.R. Acad. Sci. Paris, 313, serie 1, pp. 411-416, 1991.

[7] I. Gasser, P.A. Markowich, B. Perthame, Dispersion and moment lemmas revisited, Hamburger Beiträge zur Angew. Math., Reihe A, preprint 139, 1998, also to appear in JDE, 1999.

[8] R.T. Glassey, The Cauchy problem in kinetic theory, Philadelphia PA, SIAM, 1996.

[9] R.T. Glassey, J. Schaeffer, The "two and one-half dimensional" relativistic Vlasov-Maxwell system, Comm. Math. Phys., 185, pp 257-284, 1997.

[10] K. Hamdache, Global existence and large time behaviour of solutions for Vlasov-Stokes equations, Japan J. Indust. Appl. Math., 15, pp 51-74, 1998.

[11] E. Horst, On the classical solutions of the initial value problem for the unmodified non-linear Vlasov equation I, Math. Meth. Appl. Sci., 3, pp 229-248, 1981.

[12] E. Horst, On the classical solutions of the initial value problem for the unmodified non-linear Vlasov equation II, Math. Meth. Appl. Sci., 4, pp 19-32, 1982.

[13] P.-E. Jabin, Large time concentrations for solutions to kinetic equations with energy dissipation, to appear in Comm. P.D.E.

[14] P.-E. Jabin, B. Perthame, Notes on mathematical problems on the dynamics of dispersed particles interacting through a fluid, to appear in N. Bellomo and M. Pulvirenti editors, Modelling in applied sciences, a kinetic theory approach, Birkhauser (1999). 
[15] P.L. Lions, B. Perthame, Lemmes de moments, de moyenne et de dispersion, C.R. Acad. Sci. Paris, 314, serie 1, pp 801-806, 1992.

[16] P.L. Lions, B. Perthame, Propagation of moments and regularity for the 3-dimensional Vlasov-Poisson System, Invent. Math., 105, pp. 415-430, 1991.

[17] B. Perthame, Higher moments for kinetic equations : application to Vlasov-Poisson and Fokker-Planck equations, Math. Meth. Appl. Sci., 13, pp 441-452, 1990.

[18] B. Perthame, Time decay, propagation of low moments and dispersive effects for kinetic equations, Comm. P.D.E., 21, pp 659-686, 1996.

[19] B. Perthame, M. Pulvirenti, Weighted $L^{\infty}$ bounds and uniqueness for the Boltzmann BGK model, Arch. Ration. Mech. Anal. 125, No. 3, pp 289-295, 1993.

[20] K. Pfaffelmoser, Global classical solutions of the Vlasov-Poisson system in three dimensions for general initial data, J. Diff. Eq., 95, pp 281-303, 1992.

[21] J. Schaeffer, Global existence of smooth solutions to the Vlasov-Poisoon system in three dimensions, Comm. P.D.E., 16, pp 1313-1335, 1991.

[22] E. M. Stein, Harmonic analysis : real variable methods, orthogonality and oscillatory integrals, Princeton University Press, 1993. 